\title{
Memory for modality: Evidence for an automatic process
}

\author{
ELYSE BRAUCH LEHMAN \\ George Mason University, Fairfax, Virginia 22030
}

\begin{abstract}
Three experiments were conducted to explore the "automatic" encoding of information about presentation modality and the use of such information during word retrieval. Children (Grades 2, 3, and 6) and adults (college students) were asked to attend to a mixed-modality (auditory and visual) list of nouns, then to recall the target words, and finally to identify the presentation modality of each word on a recognition list. Instructions (incidental vs. intentional), list length, and list organization (unrelated words vs. words from taxonomic categories) were varied across the experiments. Although these manipulations affected the recall of target words, they did not change the amount of modality information retained, which was clearly above chance in all three experiments. As predicted by the Hasher and Zacks (1979) model for automatic processing, there were no developmental changes on memory for modality, instructions to remember modality information had no effect on modality identification, and a tradeoff between word recall and modality identification rarely occurred.
\end{abstract}

It is a common experience when attempting to retrieve facts to note that they were heard in a lecture or that they were read in a text. Memory for presentation modality has also been clearly demonstrated with both adults and children in laboratory studies in which accuracy levels for modality information often exceed $70 \%$ (Bray \& Batchelder, 1972; Hintzman, Block, \& Inskeep, 1972; Kirsner, 1974; Lehman \& Hanzel, 1981; Madigan \& Doherty, 1972). Although Underwood (1969) first raised the issue of modality attributes in memory in 1969 , many questions still remain to be answered about how such information is processed. Although studies suggest that memory for modality is an automatic process and that modality information can serve as a retrieval cue, there are inconsistencies and gaps in the studies that need to be addressed before more definitive answers can be given. A more concerted attempt to understand such processing seems warranted, given the emphasis placed on modality effects in studies on short-term memory (Penney, 1975).

Work on the automaticity question in general has become considerably more focused since the publication of Hasher and Zacks' (1979) seminal paper. They pro-

This research was supported by a university research grant to the author from George Mason University. Portions of this paper were presented at meetings of the Eastern Psychological Association, Baltimore, Maryland, April 1982. I wish to thank Sister Lynmarie, principal, and the teachers, parents, and students of St. Leo's School, Fairfax, Virginia, for their participation in this study. Jim Mikesell, Sharron Hanzel, and Edythe Wiggs provided enthusiastic and competent help with various phases of the study, and I am most grateful to them. Requests for reprints should be addressed to the author at the Department of Psychology, George Mason University, 4400 University Drive, Fairfax, Virginia 22030. posed a distinction between the automatic memory processes that claim minimal attention and the more strategy-dependent, or "effortful," memory processes that require maximal attention, and they listed five criteria that could be used to distinguish between the two processes. Theoretically, effortful, but not automatic, processes should be affected by manipulations within each criterion. An automatic process, for example, should occur as readily under incidental and intentional learning conditions, should not be affected by practice, should produce minimal disruption to other processes going on at the same time, should not be altered under conditions of stress, and should show few developmental changes. Although presentation modality was not specifically listed by Hasher and Zacks as an attribute that is processed automatically, the results of other studies suggest that it may be and that it should be added to Hasher and Zacks' list of automatically encoded attributes that includes space, time, and frequency of occurrence. It satisfies the criterion of equal learning under incidental and intentional conditions in both adults and children (Bray \& Batchelder, 1972; Lehman \& Hanzel, 1981) and appears at a high level even in young children (Lehman \& Hanzel, 1981). In contrast, data on the criterion of interference between operations are mixed. A tradeoff between item and modality information when subjects attempted to learn both was found by Bray and Batchelder (1972) and Madigan and Doherty (1972) in adults, but not by Lehman and Hanzel (1981) in groups of children and adults. No data presently exist on the remaining two criteria.

Retrieval questions have also become a focus of study since Hasher and Zacks (1979) first speculated that one of the cognitive functions of automatically encoded 
information is to guide the retrieval process. For example, what is the relationship between modality information and other stored attributes, such as word meaning? If it is true that modality information can serve as a retrieval cue, then one might expect that modality and item information would be stored as a unit, and remembered or forgotten together (Hasher \& Zacks, 1979). Experimental results, however, are contradictory. Bray and Batchelder (1972) found no relationship; Hintzman et al. (1972) reported that for adults the identification of modality was better for words that had been recalled, at least visually presented words; and Lehman and Hanzel (1981) observed a tendency for this relationship to be stronger for adults than for children.

We would also like to know how modality information is used during word retrieval, but, again, the results are somewhat mixed. Although organizing recall by modality (i.e., auditorily presented words in one category, visually presented words in another) has been observed for adults in several studies (Hintzman et al., 1972; Lehman \& Hanzel, 1981; Murdock \& Walker, 1969; Nilsson, 1973), it did not occur for adults in Bray and Batchelder's (1972) study or for children in Lehman and Hanzel's (1981) study. Yet to be explored is how likely presentation modality is to be used as the basis for a retrieval plan when other types of organization (e.g., taxonomic) are present.

The purpose of the present series of studies was to investigate three of Hasher and Zacks' (1979) criteria (developmental trends, intentional vs. incidental learning, and interference among operations) in greater depth in order to account for the inconsistencies and the gaps discussed above. The results will more clearly locate the modality attribute on the proposed continuum from automatic to effortful memory processes and provide data on the use of modality information during word retrieval. Instructions, list length, and list organization were varied across three experiments. Theoretically, if memory for modality occurs automatically, these manipulations should have no effect on the retention of modality information.

A modification of the Bray and Batchelder (1972) procedure was used in which subjects were asked to attend to a mixed-modality list of words. Sometimes they were told in advance to remember the input mode, sometimes they were instructed to learn the words alone, and sometimes the directions did not include any information about the memory tasks to follow. Memory for modality was measured in two ways: (1) identification of presentation modality on a final recognition test and (2) clustering by mode of input in free recall. Although the data were collected at the same time in one school by the same experimenters, they will be presented as three separate experiments because they examined different questions. Cross-experiment comparisons will, however, be discussed.

\section{EXPERIMENT 1}

In Experiment 1, children and adults were given a task that ensured that attention would be given to the list of words, without any requirement to remember either the words or their presentation modality. Whereas in prior experiments it has been only memory for modality that was incidental (standard incidental), in the present experiment it is memory for presented words, as well as for modality, that is incidental. This true incidental condition was suggested by Mandler, Seegmiller, and Day (1977) as a means of controlling for the possibility that subjects given standard incidental instructions could deliberately use the "incidental" information as a mnemonic device for remembering the words, with the result that they remembered as much about modality as when they were instructed to remember both kinds of information (intentional). Thus, a condition in which the input mode is not actively processed is essential to assess Hasher and Zacks' (1979) criterion of equal learning of modality information under incidental and intentional conditions. The true incidental condition is also necessary for evaluating the possibility that this functional equivalence of standard incidental and intentional conditions, in which subjects deliberately attempt to learn both kinds of information, produced the lack of a tradeoff between item and modality information in the Lehman and Hanzel (1981) paper. It is an important point considering the fact that both Bray and Batchelder (1972) and Madigan and Doherty (1972) argued for some interference among operations in comparisons involving only intentional and standard incidental conditions.

\section{Method}

Subjects. Sixteen second-graders and 16 sixth-graders who were attending a suburban parochial school were tested when their parental permission slips were returned. In addition, 18 college students participated in order to receive extra credit in introductory psychology courses. Two college students were subsequently eliminated, however, because debriefing indicated that they had correctly guessed the true focus of the study. All final groups were composed of an equal number of males and females and were balanced with respect to presentation lists.

Materials. The materials were those used in the Lehman and Hanzel (1981) study. Briefly, 32 nouns from first-grade readers were presented in a mixed-mode manner on videotape equipment (i.e., 16 visually and 16 auditorily). A $4 \mathrm{sec}$ interval occurred from the beginning of one presentation to the next. Each visual word was exposed for $2 \mathrm{sec}$, each auditory word for necessarily less. No more than three words in the same modality appeared in succession, and an attempt was made to separate words with obvious associations. Two lists of target words were prepared. Although the same 32 words appeared in the same order in each list, the presentation modality of each word differed between lists. Practice lists consisting of six one- and twodigit numbers were also designed in the same manner.

The modality identification list was constructed by randomly combining the 32 target words with 32 new (distractor) nouns. Each one was typed in uppercase letters on a white 3 in. $x 5$ in. card. The same order was used for all subjects. 
Procedure. Subjects were tested individually. They were asked first to make a judgment about each target word as it was presented, then to recall the target words, and finally to identify the presentation modality of each word on the modality identification list. The judgment task consisted of indicating which words signified something that could be eaten. No mention was made of the recall and recognition tasks to come, but the number practice lists were presented to give subjects some idea of how and how quickly the target words would appear. To make the procedure more believable for the adults, they were told that the task had been designed primarily for young children, but that comparison data with older groups were needed in order to study developmental trends.

As much time as was necessary was given for the spoken recall of the words. When the subject stated that no other words could be recalled, the modality identification task was administered. Each of the 64 words in this set was both shown and read aloud by the experimenter, and subjects were required to indicate whether the word had been "seen before, heard before, or was brand new" (i.e., not presented). The task was again subject-paced. Subjects also rated their confidence in the modality judgments on a 3 -point scale. These confidence ratings were not analyzed, however, and will not be discussed further.

\section{Results}

Modality identification. The left panel of Figure 1 presents the conditional proportions of correct modality identification, given word recognition for all 16 visual and 16 auditory target words. That is, of those words recognized as having been presented before, for how many was the presentation modality also correctly identified? These proportions not only allow the separation of occurrence and modality judgments, but also provide some control over age-related differences in response bias. Although the false alarm rates to the distractor words (i.e., incorrect "old" judgments) were quite low overall (see Table 1), there were age differences that suggested that the simple proportions (e.g., the number of times a subject said "visual" divided by the number of visually presented words) of the second graders might be artificially inflated, since these children were somewhat
Table 1

Hit, False Alarm, and Auditory Bias Rates

\begin{tabular}{lccc}
\hline & Hits & False Alarms & Auditory Bias \\
\hline & \multicolumn{3}{c}{ Experiment 1 } \\
Grade 2 & .85 & .13 & .57 \\
Grade 6 & .91 & .06 & .76 \\
College & .93 & .10 & .66 \\
& & Experiment 2 & \\
Grade 3 & .81 & .07 & .64 \\
College & .89 & .04 & .90 \\
& & Experiment 3 & \\
Grade 2 & .71 & .11 & .79 \\
Grade 6 & .79 & .09 & .55 \\
College & .88 & .10 & .46 \\
\hline
\end{tabular}

more likely to label a new word as "old." The proportion of hits (correct "old" judgments), which were quite high for all age groups, are also presented in Table 1 .

Figure 1 indicates a high level of modality identification at all ages, with minimal developmental change (mean modality identification for Grades 2, 6, and college $=.78, .87$, and .84 , respectively). Grade differences were not statistically reliable in a 3 (grade) by 2 (modality) ANOVA with repeated measures on the last factor $[F(2,45)=2.06, p>.10]$. Inspection of the data revealed that the apparently lower performance of the second-graders was due entirely to two children who obtained very low scores. With their scores removed, the mean for the second-graders was .82. The ANOVA also indicated that whether a word was presented visually or auditorily had no effect on modality identification (both main and interaction $F_{S}<1$ ). List and sex were not included in the modality identification ANOVA because preliminary $t$ tests showed no differences between List $A$ and List $B$ or between males and females (both ts $<1$ ).

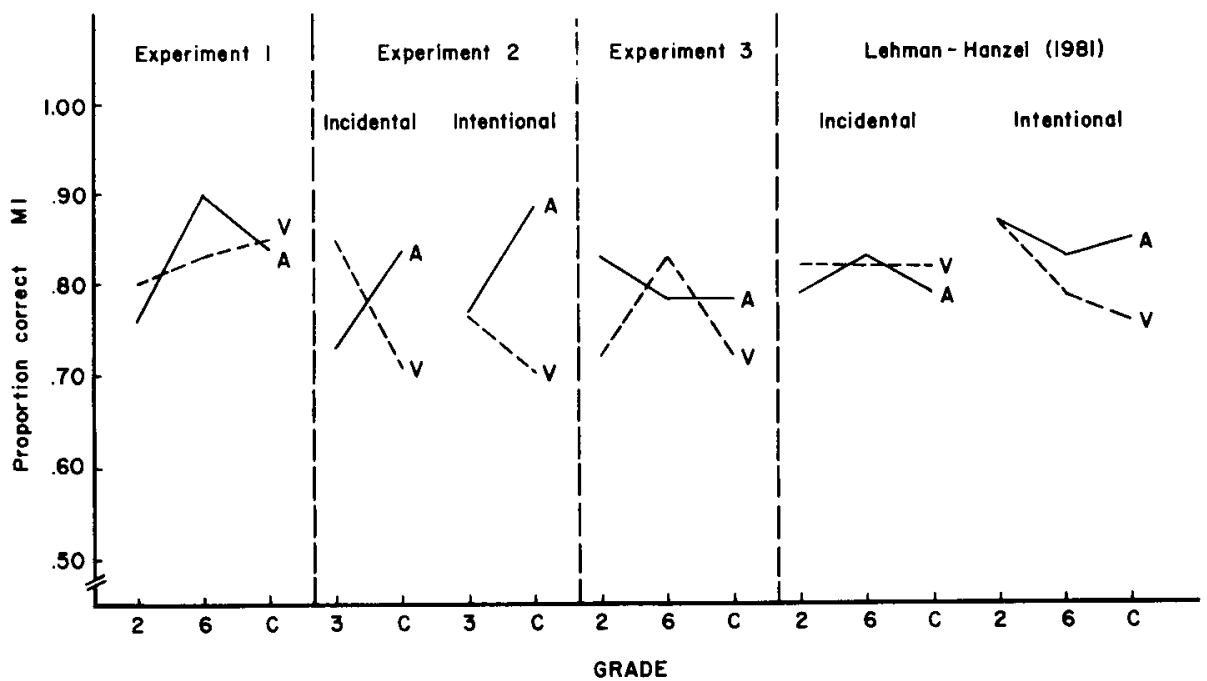

Figure 1. Conditional proportions of correct modality identification (MI), given word recognition for visual $(V)$ and auditory (A) target words by Grades 2, 3,6, and college (C) in Experiment 1 (true incidental instructions), Experiment 2 (reduction to 16 words), Experiment 3 (taxonomically categorized words), and the Lehman and Hanzel (1981) study (32 unrelated words). 
All of the observed modality identification rates exceeded what would be expected on the basis of chance. Guessing rates were calculated separately for each grade and modality following Bray and Batchelder's (1972) procedures. The method involves splitting the proportion of target words judged "old" on the modality identification task both in half and according to the auditory bias, which is calculated by dividing the auditory false alarm rate by the sum of the auditory and visual false alarm rates for the distractor words. When the observed proportions were compared with their appropriate guessing rates, all were significantly higher than chance, whether or not the guessing rates took in to account the auditory bias (see Table 1). The closest to chance were the sixth-graders identifying auditorily presented words (observed rate $=.90$; guessing rate for that condition according to the auditory bias calculation $=.69)$. A t test showed that even this difference was statistically reliable $[\mathrm{t}(15)=11.61, \mathrm{p}<.001]$.

Finally, subjects in the true incidental condition identified presentation modality as well as those in both the standard incidental and the intentional conditions of the Lehman and Hanzel (1981) study (means $=.83, .81$, and .84 , respectively). This comparison can be seen in Figure 1. A 3 (grade) by 3 (instructions) ANOVA revealed no significant differences on modality identification scores between the three instructions or between grades (both Fs $<1$ ). The Lehman and Hanzel data were collected by two of the experimenters from the present study. Target words and presentation methods were identical in both studies. Although two different parochial schools participated, they served very similar neighborhoods in the same county in northern Virginia.
Word recall and its relationship to modality identification. In contrast to the high level of modality identification at all ages, dramatic increases occurred across the grades on word recall. These changes can be seen in the first panel of Figure 2. A 3 (grade) by 2 (modality) repeated-measures ANOVA documented both these increases with age $[F(2,45)=13.19, p<.001]$ and also the better recall of visually, as opposed to auditorily, presented words $[\mathrm{F}(1,45)=10.98, \mathrm{p}<.005]$. The interaction was not statistically significant $(F<1)$. Again, preliminary $t$ tests found no differences on recall scores between List A and List B or between males and females (both ts $<1$ ).

The recall scores in this true incidental experiment were lower than those in both the standard incidental and intentional conditions of the Lehman and Hanzel (1981) study (means $=9.42,11.44$, and 10.60 , respectively). A 3 (grade) by 3 (instructions) ANOVA showed statistically significant main effects of grade $[F(2,135)=$ $57.82, \mathrm{p}<.001]$ and instructions $[\mathrm{F}(2,135)=5.07$, $p<.01]$. Newman-Keuls tests on the instructions main effect indicated that the standard incidental and intentional conditions did not differ significantly from each other, but that the true incidental condition was reliably inferior to standard incidental instructions $(p<.01)$ and tended to be inferior to intentional instructions $(p<.10)$.

Finally, there was no evidence of higher modality identification for words that had been recalled. The proportions of correct identification of modality are presented in Table 2 separately for words recalled and for words not recalled. For the initial grade by recalled/not recalled ANOVA, scores were pooled over presentation modality because of the relatively small number of

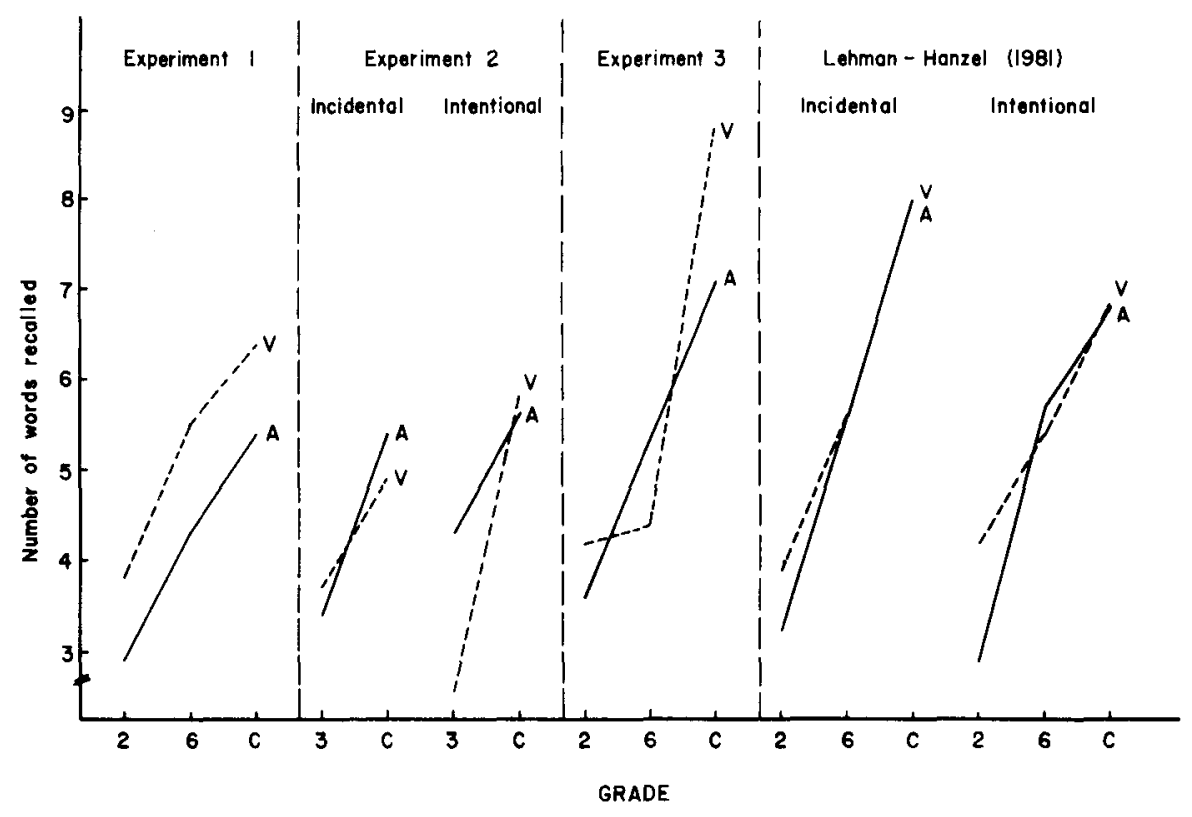

Figure 2. Mean number of visual (V) and auditory (A) target words recalled by Grades 2, 3, 6, and college (C) in Experiment 1 (true incidental instructions), Experiment 2 (reduction to 16 words), Experiment 3 (taxonomically categorized words), and the Lehman and Hanzel (1981) study (32 unrelated words). 
Table 2

Modality Identification for Words Recalled (R) and Words Not Recalled (NR)

\begin{tabular}{|c|c|c|c|c|c|c|}
\hline & \multicolumn{2}{|c|}{ Auditory Words } & \multicolumn{2}{|c|}{ Visual Words } & \multicolumn{2}{|c|}{ All Words } \\
\hline & $\mathbf{R}$ & NR & $\mathbf{R}$ & NR & $\mathbf{R}$ & NR \\
\hline & \multicolumn{6}{|c|}{ Experiment 1} \\
\hline $\begin{array}{l}\text { Grade } 2 \\
\text { Grade } 6 \\
\text { College }\end{array}$ & $\begin{array}{l}.69 \\
.90 \\
.84\end{array}$ & $\begin{array}{l}.79 \\
.90 \\
.84\end{array}$ & $\begin{array}{l}.66 \\
.84 \\
.86\end{array}$ & $\begin{array}{l}.84 \\
.82 \\
.84\end{array}$ & $\begin{array}{l}.70 \\
.86 \\
.85\end{array}$ & $\begin{array}{l}.78 \\
.87 \\
.83\end{array}$ \\
\hline & \multicolumn{6}{|c|}{ Experiment 2-Incidental } \\
\hline \multirow[t]{2}{*}{$\begin{array}{l}\text { Grade } 3 \\
\text { College }\end{array}$} & $\begin{array}{l}.62 \\
.83\end{array}$ & $\begin{array}{l}.77 \\
.91\end{array}$ & $\begin{array}{l}.91 \\
.78\end{array}$ & $\begin{array}{l}.77 \\
.58\end{array}$ & $\begin{array}{l}.76 \\
.80\end{array}$ & $\begin{array}{l}.77 \\
.75\end{array}$ \\
\hline & \multicolumn{6}{|c|}{ Experiment 2-Intentional } \\
\hline \multirow[t]{2}{*}{$\begin{array}{l}\text { Grade } 3 \\
\text { College }\end{array}$} & $\begin{array}{l}.75 \\
.89\end{array}$ & $\begin{array}{l}.84 \\
.88\end{array}$ & $\begin{array}{l}.82 \\
.69\end{array}$ & $\begin{array}{l}.70 \\
.82\end{array}$ & $\begin{array}{l}.79 \\
.79\end{array}$ & $\begin{array}{l}.77 \\
.85\end{array}$ \\
\hline & \multicolumn{6}{|c|}{ Experiment 3} \\
\hline $\begin{array}{l}\text { Grade } 2 \\
\text { Grade } 6 \\
\text { College }\end{array}$ & $\begin{array}{l}.80 \\
.72 \\
.71\end{array}$ & $\begin{array}{l}.84 \\
.82 \\
.82\end{array}$ & $\begin{array}{l}.76 \\
.85 \\
.76\end{array}$ & $\begin{array}{l}.69 \\
.82 \\
.60\end{array}$ & $\begin{array}{l}.79 \\
.77 \\
.75\end{array}$ & $\begin{array}{l}.76 \\
.82 \\
.72\end{array}$ \\
\hline
\end{tabular}

Table 3

Modality and Taxonomic Category Clustering in Recall

Modality Clustering Taxonomic Clustering

Experiment 1

\begin{tabular}{lll} 
Grade 2 & -.166 \\
Grade 6 & -.186 & \\
College & -.200 & \\
& \multicolumn{2}{c}{ Experiment 2 } \\
Grade 3 & -.343 & \\
College & $-.432^{*}$ & \\
& & Experiment 3 \\
Grade 2 & -.256 & $-.619^{*}$ \\
Grade 6 & -.134 & $-1.029^{* *}$ \\
College & +.317 & $-1.315^{* *}$ \\
\hline
\end{tabular}

* $p<.05 . \quad * * p<.01$.

words recalled by the children. In this analysis of all words, neither the main effect of recalled/not recalled nor its interaction with age reached significance $[\mathrm{F}<1$ and $F(2,45)=1.02, p>.10$, respectively]. Subsequently, the auditory and visual words were analyzed separately. These means can also be seen in Table 2 . The analysis for the visually presented words produced a reliable Grade by Recalled/Not Recalled interaction $[F(2,45)=3.58, \quad p<.05]$. Simple effects analyses revealed that a difference between recalled and not recalled words occurred only for second-graders $[F(1,45)=8.42, p<.01 ; F s$ for other grades $<1]$. They did worse when identifying the modality of words they had recalled. Although inspection of the means for the auditorily presented words suggested the same effect, the statistical analysis indicated that the difference between recalled and not recalled words was not reliable (for both main effect and interaction with grade, $\mathrm{Fs}<1$ ).

Recall order. There was little evidence of any attempt to organize recall by presentation modality in any age group. Auditory and visual presentations were treated as two categories, and Frankel and Cole's (1971) z score was calculated for each subject. These scores are presented in Table 3. A score of 0 indicates no clustering, and the higher the negative score, the greater the amount of organization by modality. None of the scores differed significantly from 0 . There were no other kinds of organization apparent in the recall lists.

\section{Discussion}

Tasks that presumably demand active processing of item information with minimal attention given to the input mode of each word still produce a remarkably high level of memory for modality. The data from this true incidental experiment indicated that retention of modality information was well above chance, even for secondgraders, and showed no improvement with increasing age. In fact, under true incidental instructions, identification of modality was as high as it had been in both conditions of the Lehman and Hanzel (1981) study. Such instructions, however, do have an effect on word recall. First, word recall was clearly lower under true than under standard incidental instructions. Second, there was no evidence of higher modality identification for words that had been recalled, as had been found by Lehman and Hanzel (1981). Third, in contrast to Lehman and Hanzel's observation that adults clustered their recall according to modality, in the present study no such organization was present in any age group.

These results corroborate Mandler et al.'s (1977) findings of lowered object recall but minimal effect on memory for spatial location of true incidental instructions. The results also indicate that even when subjects do not expect to be asked to remember a list of words and thus are less likely to use presentation modality as an aid to object recall, they still remember a great deal of information about modality.

\section{EXPERIMENT 2}

In Experiment 2 the target list presented to children and adults was reduced by half in order to increase the possibility of observing developmental change in the retention of modality information. As discussed by Kail (1979), when the number of stimuli to be remembered is large, it will be difficult to find appropriate storage strategies, and developmental change will, therefore, be negligible. On the other hand, with a small number of stimuli, mnemonic strategies, such as organization by modality, should be easier to accomplish. Since older children and adults are more likely than young children to spontaneously utilize strategies (Moely, Olson, Halwes, \& Flavell, 1969), reduction of the target list might produce age changes in memory for modality. Studies of other proposed automatic processes (e.g., spatial location and time) that do show changes with age when small groupss of to-be-remembered objects are used (Mandler et al., 1977; Mathews \& Fozard, 1970; 
von Wright, Gebhard, \& Karttunen, 1975) support such an approach. The list manipulation thus deals primarily with Hasher and Zacks' (1979) developmental change criterion. The experiment also provides a check on two other Hasher and Zacks' criteria. Since it includes both standard incidental and intentional conditions, the generality of Lehman and Hanzel's (1981) findings of no effect of instructional conditions on modality identification and no interference with other memory operations can be explored.

\section{Method}

Subjects. Thirty-two third-graders and 32 college students were obtained from the same schools that participated in Experiment 1 . Half of the subjects in each age group were given standard incidental instructions and the other half received intentional instructions. Each group was completely balanced with respect to sex and lists.

Materials. The two target lists were prepared as in Experiment 1 , except that each list contained only 16 words selected randomly from the original 32-word list. Eight were presented visually and eight auditorily in a mixed-mode manner. The practice lists of numbers were the two used in Experiment 1. Sixteen words randomly selected from the original 32 distractor items were combined with the 16 target words to form the modality identification list. These 32 words were typed individually on cards, as previously described.

Procedure. Subjects were tested individually with a procedure that involved (1) practice with numbers, (2) presentation of the target words, (3) recall of the target words, and (4) a recognition test of modality information. During presentation of the target list, all subjects were told that they would sometimes see and sometimes hear words, and that they were to remember as many words as possible. Those in the intentional condition were further directed to remember the modality of presentation. During practice with numbers, this group was asked to indicate which of the recalled numbers had been seen and which had been heard before. Retention of modality information, on the other hand, was not mentioned to those in the incidental condition until the final recognition test.

\section{Results}

Modality identification. Figure 1 (Panel 2), which presents the conditional proportions of correct modality identification, given word recognition for visual and for auditory target words, revealed again the high level of retention of information about presentation modality at all ages and the lack of overall developmental change (mean modality identification for Grade 3 and college = .78). In a 2 (grade) by 2 (instructions) by 2 (modality) ANOVA with repeated measures on the last factor, there were no statistically significant main effects on modality identification scores [ $F_{S}<1$ for grades and instructions; $F(1,60)=1.94, p>.10$ for modality]. In both Grade 3 and college groups, subjects retained as much information about modality whether they were specifically requested to or not. Only the Modality by Grade interaction was statistically significant $[F(1,60)=8.69$, $\mathrm{p}<.005]$. This interaction reflects the fact that adults had higher modality identification scores for auditory than for visual words $[F(1,60)=9.48, p<.005]$, whereas there was no reliable modality difference for the third grade $[F(1,60)=1.25, p>.10]$. The means for adults were .86 and .71 (auditory and visual, respectively); the means for children were .75 and .81 . It seems likely that the modality effect at the college level can be attributed to a large auditory bias, since this group was more likely to report auditory than visual stimuli (see Table 1). Preliminary $t$ tests showed no effect on modality identification from either list or $\operatorname{sex}[\mathrm{t}(62)=1.15, \mathrm{p}>.10 ; \mathrm{t}<1]$.

When the observed proportions were compared with their appropriate guessing rates, calculated according to Bray and Batchelder's (1972) procedure, all but one were significantly higher than chance. The nonsignificant case was for the adults in the incidental auditory condition, for which chance was calculated according to the auditory bias method. The large auditory bias produced a guessing rate for this condition of .80 , which was compared with an observed proportion of .84. This observed rate did, however, exceed chance when the guessing rate was calculated according to the no-bias method $[t(15)=11.07, p<.001]$. The condition that showed the second smallest difference from the auditory-bias guessing rate was auditory intentional for adults. Their observed modality identification rate of .89 differed reliably from a guessing rate of $.81[\mathrm{t}(15)=2.12, \mathrm{p}<.05]$.

Finally, the identification of modality when the target list contained only 16 words (Experiment 2) was at the same high level for both children and adults as when the target list contained 32 words (Lehman \& Hanzel, 1981). The overall means were .78 and .83 , respectively. A 2 (grade) by 2 (instructions) by 2 (experiment) ANOVA produced no statistically significant main effects or interactions [all $\mathrm{Fs}<1$, except main effect of experiment, $F(1,120)=3.49, p<.10$, and three-way interaction, $F(1,120)=1.21, p>.10]$.

Word recall and its relationship to modality identification. The second panel of Figure 2 again shows the large improvement with age in the number of target words recalled. A 2 (grade) by 2 (instructions) by 2 (modality) ANOVA with repeated measures on the last factor documented this grade change $[F(1,60)=69.24$, $\mathrm{p}<.001]$. Although no main effects of instructions or modality were obtained $[F<1$ and $F(1,60)=3.85$, $\mathrm{p}<.10$ ], there was a statistically significant three-way interaction $[F(1,60)=8.12, p<.01]$. Simple effects analyses revealed that third-graders recalled fewer words under intentional than incidental instructions for the visually presented words only $[F(1,120)=6.09$, $p<.025]$. The difference between instructional conditions did not reach significance for any other grade by modality combination [Grade 3 auditory, $F(1,120)=$ $3.30, \mathrm{p}<.10$; adults auditory, $\mathrm{F}<1$; adults visual, $F(1,120)=3.79, p<.10]$. Preliminary $t$ tests found no differences on recall scores between List $A$ and List $B$ or between males and females $[\mathrm{t}(62)=1.34, \mathrm{p}>.10$, and $\mathrm{t}(62)=1.15, \mathrm{p}>.10$, respectively $]$.

The proportion of words recalled on the 16-word target list was considerably higher than it had been on 
Lehman and Hanzel's (1981) 32-word target list. Means were .56 and .35 , respectively. A 2 (grade) by 2 (instructions) by 2 (experiment) ANOVA showed reliable effects of grade $[F(1,120)=131.62, p<.001]$, experiment $[F(1,120)=102.10, p<.001]$, and the threeway interaction $[F(1,120)=4.72, p<.05]$. The interaction reflects the fact that the strength of the difference between experiments in proportion recalled varied with grade and instructions. The difference was, however, statistically significant for all grade by instructions combinations [Grade 3 incidental, $F(1,120)=28.50$, $\mathrm{p}<.001$; Grade 3 intentional, $\mathrm{F}(1,120)=19.75, \mathrm{p}<.001$; adults incidental, $F(1,120)=12.27, p<.001$; adults intentional, $F(1,120)=48.06, p<.001]$.

As in the previous experiment, prior recall of a word had no reliable effect on modality identification when all words were considered (see Table 2). No statistically significant differences appeared in this 2 (grade) by 2 (instructions) by 2 (recalled/not recalled) ANOVA with repeated measures on the last factor (all $\mathrm{Fs}<1$, except recall by instructions, $\mathrm{F}(1,60)=1.68, \mathrm{p}>.10$, and recall by instructions by grade, $F(1,60)=3.11$, $\mathrm{p}<.10]$. However, a different pattern emerged when the results were analyzed separately for each presentation modality. The ANOVA on the visual words produced a significant main effect of recalled/not recalled $[F(1,60)=4.71, p<.05]$, as well as a significant interaction between recalled/not recalled and instructions $[F(1,60)=5.96, p<.05]$. This interaction reflects the fact that the difference between recalled and not recalled words was reliable for the incidental condition $[F(1,60)=10.69, p<.005]$ but not for the intentional condition $(F<1)$. Thus, both children and adults clearly showed better identification of modality for words that had been recalled than for words that had not, at least when the words were presented visually. For the words presented auditorily, on the other hand, whether or not a word had been recalled had no reliable effect on the identification of its modality As in Experiment 1, although Table 2 suggests somewhat lower modality identification for recalled words, the analysis produced only a trend toward a significant main effect of recalled/ not recalled $[F(1,60)=3.27, p<.10]$ and no significant interactions with it [all $F_{s}<1$, except the interaction with grade, $F(1,60)=1.49, p>.10]$.

Recall order. Table 3 presents the Frankel and Cole (1971) $\mathrm{z}$ scores for clustering recall by modality. Although the grade difference was not statistically reliable $(t<1)$, the scores of the adults were significantly different from no clustering $[\mathrm{t}(31)=1.97, \mathrm{p}<.05]$. The clustering scores of the children, however, did not differ reliably from zero $[t(31)=1.61, p<.10]$.

\section{Discussion}

Reducing the target list by half had no overall effect on modality identification. Retention of modality information was no higher on the shorter list than it had been on the longer list for either children or adults, although a very large auditory bias in the adult group in Experiment 2 made it more difficult to demonstrate the adults' high levels of accuracy on the modality identification task. In addition, identification of presentation modality was again not affected by instructions to remember such information.

The list length manipulation, however, did have an effect on word recall. With the shorter list, the proportion of words recalled increased, and there was some evidence for a tradeoff between memory for a word and its modality. At least for visually presented words, when third graders were asked to remember both kinds of information, their word recall was lower than when they were instructed to remember the words alone. Not all aspects of recall were as affected, however. As found previously by Lehman and Hanzel (1981), the college students were the only group that showed any reliable evidence of clustering by modality. There was also some support for Lehman and Hanzel's finding that prior recall of a word increased the probability of identification of its modality, at least for visually presented words in the incidental condition. However, no statistically reliable relationship between word recall and modality identification appeared for the auditory words, and the relationship did not interact with grade in any analysis.

These results suggest that the list manipulation had an effect on the strategies used by the children but not on those used by the adults. The adults were already, to a certain extent, spontaneously organizing recall according to modality on the longer list used by Lehman and Hanzel (1981) and may have needed stronger instructions in order to increase their clustering by modality (Nilsson, 1973). The third-graders, on the other hand, seemed to select an inefficient strategy. They still did not reliably organize their recall according to modality, yet they recalled fewer visually presented words when asked to remember both the words and their modality than when instructed to remember the words only. The effects on word recall that were produced by changes in the length of the list of target words contrast with the lack of an effect on modality identification. Taken together, they demonstrate that both children and adults remember a great deal of information about modality whether or not they make an effort to remember such information.

\section{EXPERIMENT 3}

Experiment 3 tested memory for modality and its use on recall tasks under standard incidental instructions with a list of words that could be organized into taxonomic categories. This manipulation was suggested by Light and Berger (1974) as another way of testing for automaticity. If memory for modality is automatic, there should be no change in modality identification for any age group as a function of the nature of the 
materials studied. Light and Berger successfully used this manipulation to demonstrate that within-modality discriminations are not automatic. They found that memory for case and color dropped to chance levels when they were uncorrelated with taxonomic category. Presenting a list of target words that calls attention to alternate organizational schemes also presents the opportunity to investigate questions about the retrieval function of automatically encoded information. One question is concerned with the likelihood of its use in a retrieval plan. It may be, for example, that presentation modality is less likely to be used as the basis for a retrieval plan when other types of organization (i.e., taxonomic) are present.

\section{Method}

Subjects. Sixteen second-graders, 16 sixth-graders, and 16 college students from the same sources utilized in the first two studies participated in Experiment 3. Each group was balanced with respect to males, females, List $A$, and List $B$.

Materials. Thirty-two nouns from first-grade reading books were selected as targets. There were four words from each of eight categories: animals, clothes, body parts, food, toys, people, vehicles, and household objects. Taxonomic category and presentation modality were uncorrelated. The nouns were presented mixed-modally on videotape, with two words from each category occurring visually and the other two auditorily. List B reversed the modality of each word on List A. The practice lists were identical to those used before.

The modality identification list was prepared by combining the 32 target words with 32 new words. These distractor words consisted 2 words from each of the target categories plus 16 unrelated words. The 64 words on the modality identification list were typed individually on cards, as described previously.

Procedure. All subjects were tested individually under standard incidental instructions. They were asked simply to remember as many target words as possible. No mention was made of modality information until after word recall, when the modality recognition test was administered.

\section{Results}

Modality identification. Information about modality was again very well retained at all age levels. This result can be seen in the third panel of Figure 1, which presents the conditional proportions of correct modality identification, given word recognition for the 16 visual and the 16 auditory target words. The overall modality identification means for Grade 2, Grade 6, and adults were $.78, .81$, and .75 , respectively. A 3 (grade) by 2 (modality) ANOVA with repeated measures on the last factor revealed no statistically significant effects on modality identification from either grade or modality $[F<1 ; F(1,45)=1.06, p>.10$; interaction $F(2,45)=$ $1.32, \mathrm{p}>.10]$. Likewise, neither list nor sex affected modality identification on preliminary $t$ tests $[t<1$; $\mathrm{t}(46)=1.53, \mathrm{p}>.10]$.

However, the modality identification proportion observed in each grade-modality combination was found to be significantly higher than chance whether or not an auditory bias was assumed. The combination closest to chance was the visual condition for adults, for which the observed value was .72 . The guessing rate, assuming auditory bias, was .47 ; the rate assuming no bias was .44 . Both comparisons were statistically significant $[\mathrm{t}(15)=$ $5.74, \mathrm{p}<.001$, and $\mathrm{t}(15)=6.56, \mathrm{p}<.001$, respectively]

Finally, the identification of modality when the target list contained words that could be categorized taxonomically was not reliably different from the identification of modality when the list could not so readily be organized into taxonomic categories. The overall means for Experiment 3 and for the standard incidental condition of Lehman and Hanzel's (1981) study were .77 and .81 , respectively. No statistically significant main effects or interactions were produced in a 3 (grade) by 2 (experiment) ANOVA on these modality identification scores [all $F s<1$, except experiment, $F(1,90)=2.14$, $\mathrm{p}>.10]$.

Word recall and its relationship to modality identification. The improvement with age in the number of target words recalled, which can be seen in the third panel of Figure 2, was confirmed in a 3 (grade) by 2 (modality) ANOVA with repeated measures on the last factor. The effect of grade was statistically significant $[F(2,45)=22.92, p<.001]$. Although the main effect of modality did not reach significance $[F(1,45)=1.24$, $p>.10]$, its interaction with grade did $[F(2,45)=3.25$, $p<.05]$. This interaction reflects the fact that the college students recalled more visual than auditory words $[F(1,45)=5.61, p<.05]$, whereas the modality difference was not significant for second- or for sixth-graders $[F<1 ; F(1,45)=1.51, p<.10]$. List and sex had no effect on recall scores in preliminary $t$ tests (both ts $<1$ ).

Figure 2 also indicates that the amount recalled was considerably higher in Experiment 3 than it was in either of the two previous experiments, especially for adults. It was, in fact, as high as it had been in the standard incidental condition of the Lehman and Hanzel (1981) study (means $=11.08$ and 11.44). When recall scores in these latter groups were compared in a 3 (grade) by 2 (experiment) ANOVA, neither the main effect of experiment nor its interaction with grade reached significance (both $F_{S}<1$ ).

Again, prior recall of a word had no reliable effect on modality identification when all words were considered (see Table 2). No statistically significant differences appeared on these overall scores in a 3 (grade) by 2 (recalled/not recalled) ANOVA with repeated measures on the last factor. However, as in the previous two experiments, when the data were analyzed separately for visually and for auditorily presented words, a relationship emerged between word recall and modality identification. For the visual words, as had been found in the incidental condition of Experiment 2, modality identification was higher for words that had been recalled than for words that had not $[F(1,45)=4.46$, $\mathrm{p}<.05]$. The effect occurred for both children and adults (Recalled/Not Recalled by Grade interaction, 
$\mathrm{F}<1)$. The results of the analysis on the auditory words also followed those of Experiment 2. Although the proportions in Table 2 suggest that higher identification of modality occurred for words not recalled, the 3 (grade) by 2 (recalled/not recalled) ANOVA produced only a trend $[F(1,45)=3.73, p<.10]$. The interaction with grade was not statistically significant $(\mathrm{F}<1)$.

Recall order. Table 3 presents the Frankel and Cole (1971) z scores for clustering recall by modality and by taxonomic category. The higher the negative score, the greater the amount of organization by modality. Ordering recall by modality did not occur reliably at any age, whereas ordering recall by taxonomic category was significantly different from the no-clustering score of 0 at all ages [Grade 2, $\mathrm{t}(15)=2.26, \mathrm{p}<.025$; Grade 6, $\mathrm{t}(15)=3.01, \mathrm{p}<.005$; adults, $\mathrm{t}(15)=2.69, \mathrm{p}<.01]$. Athough Table 3 suggests that taxonomic category clustering increased with age, a one-way ANOVA indicated that the grade effect was not statistically reliable $[F(2,45)=1.79, p>.10]$. There was, however, significantly more category than modality clustering in all groups. A 3 (grade) by 2 (type of clustering) ANOVA with repeated measures on the last factor produced a statistically significant effect of type of clustering $[F(1,45)=12.15, p<.005]$, but no effect of grade or any interaction between clustering and grade $[\mathrm{F}<1$; $F(2,45)=1.77, p>.10]$.

\section{Discussion}

Even with a list that made it likely that words would be organized according to taxonomic category, retention of modality information was again high. Word recall was also as high as it had been in the standard incidental condition of the Lehman and Hanzel (1981) paper and was related to modality identification for visually presented words. In contrast, clustering of recall by modality was negligible at all ages, whereas organizing recall according to taxonomic category was performed by both children and adults.

Taken together with Light and Berger's (1974) finding that case and color attributes are not selected for storage when the subject is concerned with taxonomic categories, the results of Experiment 3 support suggestions that between-modality discriminations are easier than within-modality discriminations (Atwood, 1971; Hintzman et al., 1972; Light and Berger, 1974). Information about presentation modality, a betweenmodality discrimination, is stored in long-term memory, even when the subject's task includes taxonomic encoding. Such incidental information is, however, apparently less likely to actually be used in the service of recall when other types of organization (i.e., taxonomic) are present.

\section{GENERAL DISCUSSION}

The results of the three experiments clearly indicate the remarkable resistance to disruption of memory for modality. A great deal of information about presentation modality is stored in long-term memory by adults and children, regardless of the memory requirements of the task, the length of the target list, or the types of encoding going on at the same time. Although these manipulations affect the recall of target words, they do not change the amount of modality information that is retained.

The evidence in the present studies provides strong support for the idea that modality encoding occurs "automatically" and suggests that the modality attribute be added to the list of attributes thought to be processed automatically. The results meet three of Hasher and Zacks' (1979) proposed criteria. First, developmental changes did not occur. Not only was modality identification high for both children and adults in the present experiments, but, in addition, the instructions and list manipulations had similar effects in all age groups. Second, there was no effect on modality identification of instructions to remember information about modality. Indeed, even in a true incidental condition, which made it unlikely that attention would be directed to presentation mode, the identification of modality was still above chance.

Third, interference between operations rarely occurred. The most direct evidence appeared in Experiment 2 , in which in three of four comparisons, the number of words recalled was the same whether or not subjects were asked to also remember presentation modality. It was only when third-graders recalled visually presented words that their memory for the words decreased under intentional learning instructions. As discussed above, the tradeoff on the shorter list may have reflected the selection of an inefficient strategy by the children. Certainly, even for second-graders, there was no reliable evidence of any interference on the longer list used by Lehman and Hanzel (1981). More indirect support for the lack of interference between operations comes from the true incidental condition in Experiment 1, which demonstrated that the lack of a tradeoff between item and modality information in the Lehman and Hanzel paper was not due to subjects in that study deliberately attending to modality information as an aid to word retrieval, even in the standard incidental condition. Thus, with the procedure used in the present studies, the bulk of the evidence favors support of Hasher and Zacks' (1970) criterion of no interference. Early reports of tradeoffs between item and modality information in adults may, in fact, reflect factors other than reallocation of attention, for example, output interference in Madigan and Doherty's (1972) experiment (Light \& Berger, 1974) or the avoidance of primacy and recency effects in Bray and Batchelder's (1972) study (Lehman \& Hanzel, 1981). Admittedly, however, support for this criterion appears quite variable, even in studies involving other "automatic" processes, such as spatial location (cf. Mandler et al., 1977; Park, Puglisi, \& Lutz, 1982; von Wright et al., 1975). A more pointed effort to direct attention might, therefore, prove to be very useful in understanding just when these tradeoffs occur.

The finding of a relationship between word recall and 
modality identification in the two studies involving memory instructions (i.e., Experiments 2 and 3 ) suggests that modality information can serve as a retrieval cue for a word. In the incidental conditions in these studies, modality identification was higher for recalled than for not-recalled words when the words had been presented visually. For auditorily presented words, on the other hand, there was a trend toward lower modality identification for recalled words. Hintzman et al.(1972) reported similar findings in their study of adults' memory for modality with incidental instructions. An explanation of the effect is not, however, clear. Hintzman et al. suggested that it was the additional visual input that occurred during written recall tests that led to confusion about original modality, and, finally, to more visual than auditory judgments given to recalled words. However, the same effect appeared in the present study, in which recall was verbal. Nor can it be attributed to the modality of presentation on the final recognition test. In the present study, these recognition words were both seen and heard, and in Hintzman et al.'s study, the effect held in a direct comparison of the two test modes.

What is clear, however, is that in the present studies there was no overall relationship between recall and modality identification, as had been found by Lehman and Hanzel (1981). Although Bray and Batchelder (1972) reported the same lack of a relationship when they analyzed across modalities, their failure to analyze separately by modality makes it impossible to know whether the combined scores masked the differential effect of modality found in the present group of studies. Lehman and Hanzel also reported a tendency for the relationship between word recall and modality identification to be stronger for adults than for children. The lack of any such interaction in the present studies at the very least points out the difficulties involved in reporting statistical trends. The experience of recalling a word seems to have the same effect on the modality identification of both children and adults. Thus, the processing of information about presentation modality is similar to the storage of information about spatial location, which shows a correlation, albeit a small one, with the ability to recall a word from long-term memory (Cumming \& Coltheart, 1969; Mandler et al., 1977) and differs from the processing of frequency information, which seems to be stored independently of other attributes (Howell, 1973).

Finally, the results raise questions about the retrieval function of modality information. They indicate that adults will spontaneously use input mode as a retrieval cue. Others have demonstrated not only this spontaneous usage with adult subjects (Hintzman et al., 1972), but also the fact that there is an additional amount of organization if adults are specifically instructed to organize the words by modality (Nilsson, 1973). Whether children can organize recall in this way under instructions is still unknown. They do not seem to reliably use input mode spontaneously as a retrieval cue. An answer would help us to understand possible developmental differences in the actual use of modality information for retrieval. The results also indicate, however, that adults do not always cluster their recall by modality and that, even when they do, it is not as strong as other types of clustering. For example, organization according to taxonomic categories took precedence over organization by modality in Experiment 3. Likewise, such organization did not take place in a situation that initially made no memory demands, that is, the true incidental condition in Experiment 1. Does this mean that modality is a weak cue, used only when nothing else is available? Does it mean that modality can be effective as a retrieval cue only if it is stored at the same time as the to-be-remembered item itself, that is, the principle of encoding specificity (Tulving \& Thomson, 1973)?

Although questions about the retrieval function of modality encoding still need to be investigated, the present series of studies has demonstrated that such encoding itself is not easily disrupted. Long-term memory representations contain a great deal of information about input mode, and the evidence is mounting that this occurs automatically.

\section{REFERENCES}

ATwoon, G. An experimental study of visual imagination and memory. Cognitive Psychology, 1971, 2, 290-299.

Bray, N. W., \& Batchelder, W. H. Effects of instructions and retention interval on memory of presentation mode. Journal of Verbal Learning and Verbal Behavior, 1972, 11, 367-374.

Cumming, G., \& Coltheart, M. Short-term memory: Are item and position information stored independently? Psychonomic Science, 1969, 15, 216-218.

Franzel, F., \& Cole, M. Measures of category clustering in free recall. Psychological Bulletin, 1971, 76, 39-44.

Hasher, L., \& Zacks, R. T. Automatic and effortful processes in memory. Journal of Experimental Psychology: General, $1979,108,356-388$.

Hintzman, D. L., Block, R. A., \& Ingkeep, N. R. Memory for mode of input. Journal of Verbal Learning and Verbal Behavior, 1972, 11, 741-749.

Howell, W. C. Storage of events and event frequencies: A comparison of two paradigms in memory. Journal of Experimental Psychology, 1973, 98, 260-263.

KAIL, R. The development of memory in children. San Francisco: Freeman, 1979.

Kursne R, K. Modality differences in recognition memory for words and their attributes. Journal of Experimental Psychology, 1974, 102, 579-584.

Lehman, E. B., \& Hanzel, S. H. A developmental study of memory for presentation modality. Journal of General Psychology, 1981, 105, 155-164.

Light, L. L., \& Berger, D. E. Memory for modality: Withinmodality discrimination is not automatic. Journal of Experimental Psychology, 1974, 103, 854-860.

Madigan, S., \& Dohenty, L. Retention of item attributes in free recall. Psychonomic Science, 1972, 27, 233-235.

Mandler, J. M., Seegmiller, D., \& DAY, J. On the coding of spatial information. Memory \& Cognition, 1977, 5, 10-16. 
Mathews, M. E., \& Fozard, J. L. Age differences in judgments of recency for short sequences of pictures. Developmental Psychology, 1970, 3, 208-217.

Moely, B. E., Olson, F. A., Halwes, T. G., \& Flavell, J. H. Production deficiency in young children's clustered recall. Developmental Psychology, 1969, 1, 26-34.

Murdock, B. B., JR., \& W AlKer, K. D. Modality effects in free recall. Journal of Verbal Learning and Verbal Behavior, $1969,8,665-676$.

NiLsson, L. Organization by modality in short-term memory. Journal of Experimental Psychology, 1973, 100, 246-253.

Park, D. C., Puglisi, J. T., \& LUTz, R. Spatial memory in older adults: Effects of intentionality. Journal of Gerontology, 1982, 37, 330-335.

Penney, C. G. Modality effects in short-term verbal memory. Psychological Bulletin, 1975, 82, 68-84.

Tulving, E., \& Thomson, D. M. Encoding specificity and retrieval processes in episodic memory. Psychological Review, $1973,80,352-373$

UNDERWOOD, B. J. Attributes of memory. Psychological Review, $1969,76,559-573$.

von Wriaht, J. M., Gebhard, P., \& KartTunen, M. A developmental study of the recall of spatial location. Journal of Experimental Child Psychology, 1975, 20, 181-190.

(Received for publication January 8, 1982; revision accepted July 28,1982 .) 